\title{
General Linear Boundary Value Problem for the Second-Order Integro-Differential Loaded Equation with Boundary Conditions Containing Both Nonlocal and Global Terms
}

\author{
M. R. Fatemi and N. A. Aliyev \\ Department of Mathematics, Baku State University, 23, Z.I. Khalilov Street, AZ1148 Baku, Azerbaijan
}

Correspondence should be addressed to M. R. Fatemi, fatemi.mehran@yahoo.com

Received 7 April 2010; Accepted 19 July 2010

Academic Editor: A. Zafer

Copyright (C) 2010 M. R. Fatemi and N. A. Aliyev. This is an open access article distributed under the Creative Commons Attribution License, which permits unrestricted use, distribution, and reproduction in any medium, provided the original work is properly cited.

The paper is devoted to obtaining the sufficient conditions for Fredholm property for the general boundary value problem of the second-order linear integro-differential equation. Here, the boundary conditions corresponding with the boundary value problem contain both nonlocal and global terms.

\section{Introduction}

As is known, the boundary value problems are studied in differential equations theory and related areas in mathematical physics with local boundary conditions for linear elliptic partial differential equations [1-4]. Also the number of boundary conditions for linear ordinary differential equation coincide with the order of the [5], and for a partial differential equation (in a bounded domain with smooth boundaries) this number coincides with half of the order of the considered [6]. Then, boundary value problems for linear ordinary differential equations sharply are different from the same problems for linear partial differential equations. Using nonlocal boundary conditions, we remove the misunderstandings given above when passing from boundary value problems for an ordinary differential equation to the problems for partial [7-9].

The investigation method is as follows. Employing fundamental solution of twodimensional Laplace equation, Green's second formula [10] and analogy of this formula [79] are constructed for the current problem. Further, necessary conditions are chosen from these formulas. Singular terms contained in necessary conditions are separated. Taking into account the fact that for the obtained singular integral equations we are on the spectrum, these singularities cannot be regularized by standard methods [11, 12]. These singularities 
are regularized by peculiar method proceeding from the given boundary conditions $[7,8]$. Finally, joining the obtained regular relations with the given boundary conditions, we get sufficient condition on Fredholm property for the stated problems.

\section{Problem Statement}

Let $D \subset R^{2}$ be a bounded convex domain with respect to $x_{2}$ with boundary $\Gamma$ supposed to be a Lyapinov line [10]. We assume that the boundary of $D$ is divided into two parts $\Gamma_{k}, k=1,2$ with the equations $x_{2}=\gamma_{k}\left(x_{1}\right), k=1,2$, and $x_{1} \in\left[a_{1}, b_{1}\right]$. So, consider the following problem:

$$
\begin{aligned}
l u \equiv & \Delta u(x)+\sum_{j=1}^{2} a_{j}(x) \frac{\partial u(x)}{\partial x_{j}}+a_{0}(x) u(x) \\
& +\left.\int_{a_{1}}^{b_{1}} \sum_{n=1}^{2}\left[\sum_{j=1}^{2} K_{j n}\left(x, \eta_{1}\right) \frac{\partial u(\eta)}{\partial \eta_{j}}+K_{0 n}\left(x, \eta_{1}\right) u(\eta)\right]\right|_{\eta_{2}=\gamma_{n}\left(\eta_{1}\right)} d \eta_{1} \\
& +\int_{D}\left[\sum_{j=1}^{2} K_{j}(x, \eta) \frac{\partial u(\eta)}{\partial \eta_{j}}+K_{0}(x, \eta) u(\eta)\right] d \eta=f(x), \\
x= & \left.x_{1}, x_{2}\right) \in D \subset R^{2}, \\
l_{m} u \equiv & \left.\sum_{n=1}^{2}\left[\sum_{j=1}^{2} \alpha_{m j n}\left(x_{1}\right) \frac{\partial u(x)}{\partial x_{j}}+\alpha_{m 0 n}\left(x_{1}\right) u(x)\right]\right|_{x_{2}=\gamma_{n}\left(x_{1}\right)} \\
& +\left.\int_{a_{1}}^{b_{1}} \sum_{n=1}^{2}\left[\sum_{j=1}^{2} A_{m j n}\left(x_{1}, \eta_{1}\right) \frac{\partial u(\eta)}{\partial \eta_{j}}+A_{m 0 n}\left(x_{1}, \eta_{1}\right) u(\eta)\right]\right|_{\eta_{2}=\gamma_{n}\left(\eta_{1}\right)} d \eta_{1} \\
& +\int_{D}\left[\sum_{j=1}^{2} A_{m j}\left(x_{1}, \eta\right) \frac{\partial u(\eta)}{\partial \eta_{j}}+A_{m 0}\left(x_{1}, \eta\right) u(\eta)\right] d \eta=\alpha_{m}\left(x_{1}\right), \\
& m=1,2, x_{1} \in\left[a_{1}, b_{1}\right],
\end{aligned}
$$

where $\Delta$ is two-dimensional Laplace operator and all the data (the coefficients, kernel of integrals, and the right-hand sides) are continuous functions. Boundary conditions (2.2) are assumed to be linearly independent.

\section{Fundamental Solution}

As is known, while investigating boundary value problems by potential theory, a fundamental solution of adjoint equation is used. If the considered equation is self-adjoint, then fundamental solution of the same equation is chosen. Taking into account that (2.1) is sufficiently general, it is very difficult to construct fundamental solution of the adjoint 
equation. Therefore, we will choose fundamental solution of the principal part of equation (2.1), that is, for two-dimensional Laplace equation as follows:

$$
U(x-\xi)=\frac{1}{2 \pi} \ln |x-\xi| .
$$

\section{Basic Relations}

At first, we construct Green's second formula connected with (2.1) and fundamental solution (3.1), that is, we multiply (2.1) by fundamental solution (3.1) and integrate it with respect to domain $D$. Further, applying Ostrogradskii-Gauss formula, we get the following:

$$
\begin{aligned}
& \int_{\Gamma}\left[u(x) \frac{d U(x-\xi)}{d v_{x}}-\frac{d u(x)}{d v} U(x-\xi)\right] d x-\sum_{j=1}^{2} \int_{D} a_{j}(x) \frac{\partial u(x)}{\partial x_{j}} U(x-\xi) d x \\
& -\int_{D} a_{0}(x) u(x) U(x-\xi) d x \\
& \quad-\int_{D}\left\{\left.\int_{a_{1}}^{b_{1}} \sum_{n=1}^{2}\left[\sum_{j=1}^{2} K_{j n}\left(x, \eta_{1}\right) \frac{\partial u(\eta)}{\partial \eta_{j}}+K_{0 n}\left(x, \eta_{1}\right) u(\eta)\right]\right|_{\eta_{2}=\gamma_{n}\left(\eta_{1}\right)} d \eta_{1}\right\} \\
& \quad \times U(x-\xi) d x-\int_{D}\left\{\int_{D}\left[\sum_{j=1}^{2} K_{j}(x, \eta) \frac{\partial u(\eta)}{\partial \eta_{j}}+K_{0}(x, \eta) u(\eta)\right] d \eta\right\} \\
& \quad \times U(x-\xi) d x+\int_{D} f(x) U(x-\xi) d x= \begin{cases}u(\xi), & \xi \in D, \\
\frac{1}{2} u(\xi), & \xi \in \Gamma,\end{cases}
\end{aligned}
$$

where $v=v_{x}$ is an external normal to the boundary $\Gamma$ of domain $D$ at the point $x \in \Gamma$.

Now, proceeding from the relations obtained in [7-9], we derive Green's second formula for (2.1) as follows:

$$
\begin{aligned}
& \int_{\Gamma} \frac{\partial u(x)}{\partial x_{1}} \frac{d U(x-\xi)}{d v_{x}} d x-\int_{\Gamma} \frac{\partial u(x)}{\partial x_{2}} \frac{d U(x-\xi)}{d \tau_{x}} d x \\
& \quad+\int_{D} \sum_{j=1}^{2} a_{j}(x) \frac{\partial u(x)}{\partial x_{j}} \frac{\partial U(x-\xi)}{\partial x_{1}} d x+\int_{D} a_{0}(x) u(x) \frac{\partial U(x-\xi)}{\partial x_{1}} d x \\
& \quad+\int_{D}\left\{\left.\int_{a_{1}}^{b_{1}} \sum_{n=1}^{2}\left[\sum_{j=1}^{2} K_{j n}\left(x, \eta_{1}\right) \frac{\partial u(\eta)}{\partial \eta_{j}}+K_{0 n}\left(x, \eta_{1}\right) u(\eta)\right]\right|_{\eta_{2}=\gamma_{n}\left(\eta_{1}\right)} d \eta_{1}\right. \\
& \left.+\int_{D}\left[\sum_{j=1}^{2} K_{j}(x, \eta) \frac{\partial u(\eta)}{\partial \eta_{j}}+K_{0}(x, \eta) u(\eta)\right] d \eta\right\} \frac{\partial U(x-\xi)}{\partial x_{1}} d x \\
& \quad-\int_{D} f(x) \frac{\partial U(x-\xi)}{\partial x_{1}} d x= \begin{cases}\frac{\partial u(\xi)}{\partial \xi_{1}} & \xi \in D, \\
\frac{1}{2} \frac{\partial u(\xi)}{\partial \xi_{1}}, & \xi \in \Gamma,\end{cases}
\end{aligned}
$$




$$
\begin{aligned}
& \int_{\Gamma} \frac{\partial u(x)}{\partial x_{1}} \frac{d U(x-\xi)}{d \tau_{x}} d x+\int_{\Gamma} \frac{\partial u(x)}{\partial x_{2}} \frac{d U(x-\xi)}{d v_{x}} d x \\
& +\int_{D} \sum_{j=1}^{2} a_{j}(x) \frac{\partial u(x)}{\partial x_{j}} \frac{\partial U(x-\xi)}{\partial x_{2}} d x+\int_{D} a_{0}(x) u(x) \frac{\partial U(x-\xi)}{\partial x_{2}} d x \\
& +\int_{D}\left\{\left.\int_{a_{1}}^{b_{1}} \sum_{n=1}^{2}\left[\sum_{j=1}^{2} K_{j n}\left(x, \eta_{1}\right) \frac{\partial u(\eta)}{\partial \eta_{j}}+K_{0 n}\left(x, \eta_{1}\right) u(\eta)\right]\right|_{\eta_{2}=\gamma_{n}\left(\eta_{1}\right)} d \eta_{1}\right. \\
& \left.+\int_{D}\left[\sum_{j=1}^{2} K_{j}(x, \eta) \frac{\partial u(\eta)}{\partial \eta_{j}}+K_{0}(x, \eta) u(\eta)\right] d \eta\right\} \frac{\partial U(x-\xi)}{\partial x_{2}} d x \\
& \quad-\int_{D} f(x) \frac{\partial U(x-\xi)}{\partial x_{2}} d x= \begin{cases}\frac{\partial u(\xi)}{\partial \xi_{2}}, & \xi \in D, \\
\frac{1}{2} \frac{\partial u(\xi)}{\partial \xi_{2}}, & \xi \in \Gamma,\end{cases}
\end{aligned}
$$

where $\tau_{x}$ are tangential directions to the boundary $\Gamma$ of domain $D$ at the point $x \in \Gamma$.

Theorem 4.1. If the data of (2.1) are continuous functions (coefficients, kernels of integrals, and the right-hand side), the domain $D$ is convex in the direction of $x_{2}$, and the boundary is Lyapinov's $\Gamma$-line, then each solution of (2.1) satisfies the basic relations (4.1)-(4.3).

Remark 4.2. The second relations obtained from (4.1)-(4.3), that is, the expressions corresponding for $\xi \in \Gamma$, are necessary conditions. Expressions (4.1)-(4.3) themselves are basic relations.

\section{Necessary Conditions}

As it was noted above, necessary conditions are obtained from relations (4.1)-(4.3). For reducing these conditions, at first we notice that

$$
\begin{array}{ll}
\frac{d U(x-\xi)}{d v_{x}} d x=\frac{1}{2 \pi\left[1+\gamma_{1}^{\prime 2}\left(\sigma_{1}\right)\right]} \cdot \frac{\gamma_{1}^{\prime}\left(\sigma_{1}\right)-\gamma_{1}^{\prime}\left(x_{1}\right)}{x_{1}-\xi_{1}} d x_{1}, & x \in \Gamma_{1}, \quad \xi \in \Gamma_{1}, \\
\frac{d U(x-\xi)}{d v_{x}} d x=\frac{1}{2 \pi\left[1+\gamma_{2}^{\prime 2}\left(\sigma_{2}\right)\right]} \cdot \frac{\gamma_{2}^{\prime}\left(\sigma_{2}\right)-\gamma_{2}^{\prime}\left(x_{1}\right)}{x_{1}-\xi_{1}} d x_{1}, \quad x \in \Gamma_{2}, \quad \xi \in \Gamma_{2}, \\
\frac{d U(x-\xi)}{d \tau_{x}} d x=\frac{1}{2 \pi\left(x_{1}-\xi_{1}\right)} \cdot \frac{1+\gamma_{1}^{\prime}\left(\sigma_{1}\right) \gamma_{1}^{\prime}\left(x_{1}\right)}{1+\gamma_{1}^{\prime 2}\left(\sigma_{1}\right)} d x_{1}, \quad x \in \Gamma_{1}, \quad \xi \in \Gamma_{1}, \\
\frac{d U(x-\xi)}{d \tau_{x}} d x=\frac{1}{2 \pi\left(x_{1}-\xi_{1}\right)} \cdot \frac{1+\gamma_{2}^{\prime}\left(\sigma_{2}\right) \gamma_{2}^{\prime}\left(x_{1}\right)}{1+\gamma_{2}^{\prime 2}\left(\sigma_{2}\right)} d x_{1}, \quad x \in \Gamma_{2}, \quad \xi \in \Gamma_{2} .
\end{array}
$$

For Lyapinov boundary, boundary values of normal derivative of fundamental solution (5.1) and (5.2) may contain only weak singularity (5.3), (5.4) and the same values of 
tangential derivative contain singularity (5.5), (5.6). If the points $x$ and $\xi$ belong to different parts of the boundary $\Gamma$, then there are no singularities in boundary values from derivative of fundamental solution (both in norms and tangential directions).

Thus, we obtain the following necessary conditions:

$$
\begin{aligned}
& \frac{1}{2} u\left(\xi_{1}, \gamma_{1}\left(\xi_{1}\right)\right)=-\frac{1}{2 \pi} \int_{a_{1}}^{b_{1}} u\left(x_{1}, \gamma_{1}\left(x_{1}\right)\right) \frac{\gamma_{1}^{\prime}\left(\sigma_{1}\right)-\gamma_{1}^{\prime}\left(x_{1}\right)}{\left[1+\gamma_{1}^{\prime 2}\left(\sigma_{1}\right)\right]\left(x_{1}-\xi_{1}\right)} d x_{1} \\
& +\frac{1}{2 \pi} \int_{a_{1}}^{b_{1}} u\left(x_{1}, \gamma_{2}\left(x_{1}\right)\right) \frac{\gamma_{2}\left(x_{1}\right)-\gamma_{1}\left(\xi_{1}\right)-\left(x_{1}-\xi_{1}\right) \gamma_{2}^{\prime}\left(x_{1}\right)}{\left(x_{1}-\xi_{1}\right)^{2}+\left[\gamma_{2}\left(x_{1}\right)-\gamma_{1}\left(\xi_{1}\right)\right]^{2}} d x_{1} \\
& -\left.\int_{a_{1}}^{b_{1}} \frac{\partial u(x)}{\partial x_{1}}\right|_{x_{2}=\gamma_{1}\left(x_{1}\right)} U\left(x_{1}-\xi_{1}, \gamma_{1}\left(x_{1}\right)-\gamma_{1}\left(\xi_{1}\right)\right) \gamma_{1}^{\prime}\left(x_{1}\right) d x_{1} \\
& +\left.\int_{a_{1}}^{b_{1}} \frac{\partial u(x)}{\partial x_{2}}\right|_{x_{2}=\gamma_{1}\left(x_{1}\right)} U\left(x_{1}-\xi_{1}, \gamma_{1}\left(x_{1}\right)-\gamma_{1}\left(\xi_{1}\right)\right) d x_{1} \\
& +\left.\int_{a_{1}}^{b_{1}} \frac{\partial u(x)}{\partial x_{1}}\right|_{x_{2}=\gamma_{2}\left(x_{1}\right)} U\left(x_{1}-\xi_{1}, \gamma_{2}\left(x_{1}\right)-\gamma_{1}\left(\xi_{1}\right)\right) \gamma_{2}^{\prime}\left(x_{1}\right) d x_{1} \\
& -\left.\int_{a_{1}}^{b_{1}} \frac{\partial u(x)}{\partial x_{2}}\right|_{x_{2}=\gamma_{2}\left(x_{1}\right)} U\left(x_{1}-\xi_{1}, \gamma_{2}\left(x_{1}\right)-\gamma_{1}\left(\xi_{1}\right)\right) d x_{1} \\
& -\sum_{j=1}^{2} \int_{D} a_{j}(x) \frac{\partial u(x)}{\partial x_{j}} U\left(x_{1}-\xi_{1}, x_{2}-\gamma_{1}\left(\xi_{1}\right)\right) d x \\
& -\int_{D} a_{0}(x) u(x) U\left(x_{1}-\xi_{1}, x_{1}-\gamma_{1}\left(\xi_{1}\right)\right) d x \\
& -\int_{D}\left\{\left.\int_{a_{1}}^{b_{1}} \sum_{n=1}^{2}\left[\sum_{j=1}^{2} K_{j n}\left(x, \eta_{1}\right) \frac{\partial u(\eta)}{\partial \eta_{j}}+K_{0 n}\left(x, \eta_{1}\right) u(\eta)\right]\right|_{\eta_{2}=\gamma_{n}\left(\eta_{1}\right)} d \eta_{1}\right\} \\
& \times U\left(x_{1}-\xi_{1}, x_{2}-\gamma_{1}\left(\xi_{1}\right)\right) d x \\
& -\int_{D}\left\{\int_{D}\left[\sum_{j=1}^{2} K_{j}(x, \eta) \frac{\partial u(\eta)}{\partial \eta_{j}}+K_{0}(x, \eta) u(\eta)\right] d \eta\right\} \\
& \times U\left(x_{1}-\xi_{1}, x_{2}-\gamma_{1}\left(\xi_{1}\right)\right) d x+\int_{D} f(x) U\left(x_{1}-\xi_{1}, x_{2}-\gamma_{1}\left(\xi_{1}\right)\right) d x
\end{aligned}
$$




$$
\begin{aligned}
& \frac{1}{2} u\left(\xi_{1}, \gamma_{2}\left(\xi_{1}\right)\right)=-\frac{1}{2 \pi} \int_{a_{1}}^{b_{1}} u\left(x_{1}, \gamma_{1}\left(x_{1}\right)\right) \frac{\gamma_{1}^{\prime}\left(x_{1}\right)-\gamma_{2}\left(\xi_{1}\right)-\left(x_{1}-\xi_{1}\right) \gamma_{1}^{\prime}\left(x_{1}\right)}{\left(x_{1}-\xi_{1}\right)^{2}+\left[\gamma_{1}\left(x_{1}\right)-\gamma_{2}\left(\xi_{1}\right)\right]^{2}} d x_{1} \\
& +\frac{1}{2 \pi} \int_{a_{1}}^{b_{1}} u\left(x_{1}, \gamma_{2}\left(x_{1}\right)\right) \frac{\gamma_{2}^{\prime}\left(\sigma_{2}\right)-\gamma_{2}^{\prime}\left(x_{1}\right)}{\left[1+\gamma_{2}^{\prime 2}\left(\sigma_{1}\right)\right]\left(x_{1}-\xi_{1}\right)} d x_{1} \\
& -\left.\int_{a_{1}}^{b_{1}} \frac{\partial u(x)}{\partial x_{1}}\right|_{x_{2}=\gamma_{1}\left(x_{1}\right)} U\left(x_{1}-\xi_{1}, \gamma_{1}\left(x_{1}\right)-\gamma_{2}\left(\xi_{1}\right)\right) \gamma_{1}^{\prime}\left(x_{1}\right) d x_{1} \\
& +\left.\int_{a_{1}}^{b_{1}} \frac{\partial u(x)}{\partial x_{2}}\right|_{x_{2}=\gamma_{1}\left(x_{1}\right)} U\left(x_{1}-\xi_{1}, \gamma_{1}\left(x_{1}\right)-\gamma_{2}\left(\xi_{1}\right)\right) d x_{1} \\
& +\left.\int_{a_{1}}^{b_{1}} \frac{\partial u(x)}{\partial x_{2}}\right|_{x_{2}=\gamma_{2}\left(x_{1}\right)} U\left(x_{1}-\xi_{1}, \gamma_{2}\left(x_{1}\right)-\gamma_{2}\left(\xi_{1}\right)\right) \gamma_{2}^{\prime}\left(x_{1}\right) d x_{1} \\
& -\left.\int_{a_{1}}^{b_{1}} \frac{\partial u(x)}{\partial x_{2}}\right|_{x_{2}=\gamma_{2}\left(x_{1}\right)} U\left(x_{1}-\xi_{1}, \gamma_{2}\left(x_{1}\right)-\gamma_{2}\left(\xi_{1}\right)\right) d x_{1} \\
& -\sum_{j=1}^{2} \int_{D} a_{j}(x) \frac{\partial u(x)}{\partial x_{j}} U\left(x_{1}-\xi_{1}, x_{2}-\gamma_{2}\left(\xi_{1}\right)\right) d x \\
& -\int_{D} a_{0}(x) u(x) U\left(x_{1}-\xi_{1}, x_{2}-\gamma_{2}\left(\xi_{1}\right)\right) d x \\
& -\int_{D}\left\{\left.\int_{a_{1}}^{b_{1}} \sum_{n=1}^{2}\left[\sum_{j=1}^{2} K_{j n}\left(x, \eta_{1}\right) \frac{\partial u(\eta)}{\partial \eta_{j}}+K_{0 n}\left(x, \eta_{1}\right) u(\eta)\right]\right|_{\eta_{2}=\gamma_{n}\left(\eta_{1}\right)} d \eta_{1}\right\} \\
& \times U\left(x_{1}-\xi_{1}, x_{2}-\gamma_{2}\left(\xi_{1}\right)\right) d x \\
& -\int_{D}\left\{\int_{D}\left[\sum_{j=1}^{2} K_{j}(x, \eta) \frac{\partial u(\eta)}{\partial \eta_{j}}+K_{0}(x, \eta) u(\eta)\right] d \eta\right\} \\
& \times U\left(x_{1}-\xi_{1}, x_{2}-\gamma_{2}\left(\xi_{1}\right)\right) d x+\int_{D} f(x) U\left(x_{1}-\xi_{1}, x_{2}-\gamma_{2}\left(\xi_{1}\right)\right) d x
\end{aligned}
$$

Theorem 5.1. Under the conditions of theorem 1, the necessary conditions (5.7) and (5.8) are regular, that is, they do not contain singular terms. 
Now, we give necessary conditions obtained from relations (4.2) and (4.3) as follows

$$
\begin{aligned}
& \left.\frac{1}{2} \frac{\partial u(\xi)}{\partial \xi_{1}}\right|_{\xi_{2}=\gamma_{1}\left(\xi_{1}\right)} \\
& =-\left.\frac{1}{2 \pi} \int_{a_{1}}^{b_{1}} \frac{\partial u(x)}{\partial x_{1}}\right|_{x_{2}=\gamma_{1}\left(x_{1}\right)} \frac{1}{1+\gamma_{1}^{\prime 2}\left(\sigma_{1}\right)} \frac{\gamma_{1}^{\prime}\left(\sigma_{1}\right)-\gamma_{1}^{\prime}\left(x_{1}\right)}{x_{1}-\xi_{1}} d x_{1} \\
& +\left.\frac{1}{2 \pi} \int_{a_{1}}^{b_{1}} \frac{\partial u(x)}{\partial x_{1}}\right|_{x_{2}=\gamma_{2}\left(x_{1}\right)} \frac{\gamma_{2}\left(x_{1}\right)-\gamma_{1}\left(\xi_{1}\right)+\left(x_{1}-\xi_{1}\right) \gamma_{2}^{\prime}\left(x_{1}\right)}{\left(x_{1}-\xi_{1}\right)^{2}+\left[\gamma_{2}\left(x_{1}\right)-\gamma_{1}\left(\xi_{1}\right)\right]^{2}} d x_{1} \\
& -\left.\frac{1}{2 \pi} \int_{a_{1}}^{b_{1}} \frac{\partial u(x)}{\partial x_{2}}\right|_{x_{2}=\gamma_{1}\left(x_{1}\right)} \frac{1+\gamma_{1}^{\prime}\left(\sigma_{1}\right) \gamma_{1}^{\prime}\left(x_{1}\right)}{1+\gamma_{1}^{\prime 2}\left(\sigma_{1}\right)} \frac{d x_{1}}{x_{1}-\xi_{1}} \\
& -\left.\frac{1}{2 \pi} \int_{a_{1}}^{b_{1}} \frac{\partial u(x)}{\partial x_{2}}\right|_{x_{2}=\gamma_{2}\left(x_{1}\right)} \frac{x_{1}-\xi_{1}+\left[\gamma_{2}\left(x_{1}\right)-\gamma_{1}\left(\xi_{1}\right)\right] \gamma_{2}^{\prime}(x)}{\left(x_{1}-\xi_{1}\right)^{2}+\left[\gamma_{2}\left(x_{1}\right)-\gamma_{1}\left(\xi_{1}\right)\right]^{2}} d x_{1} \\
& +\left.\int_{D} \sum_{j=1}^{2} a_{j}(x) \frac{\partial u(x)}{\partial x_{j}} \frac{\partial U(x-\xi)}{\partial x_{1}}\right|_{\xi_{2}=\gamma_{1}\left(\xi_{1}\right)} d x \\
& +\left.\int_{D} a_{0}(x) u(x) \frac{\partial U(x-\xi)}{\partial x_{1}}\right|_{\xi_{2}=\gamma_{1}\left(\xi_{1}\right)} d x \\
& +\int_{D}\left\{\left.\int_{a_{1}}^{b_{1}} \sum_{n=1}^{2}\left[\sum_{j=1}^{2} K_{j n}\left(x, \eta_{1}\right) \frac{\partial u(\eta)}{\partial \eta_{j}}+K_{0 n}\left(x, \eta_{1}\right) u(\eta)\right]\right|_{\eta_{2}=\gamma_{n}\left(\eta_{1}\right)} d \eta_{1}\right. \\
& \left.+\int_{D}\left[\sum_{j=1}^{2} K_{j}(x, \eta) \frac{\partial u(\eta)}{\partial \eta_{j}}+K_{0}(x, \eta) u(\eta)\right] d \eta\right\}\left.\frac{\partial U(x-\xi)}{\partial x_{1}}\right|_{\xi_{2}=\gamma_{1}\left(\xi_{1}\right)} d x \\
& -\left.\int_{D} f(x) \frac{\partial U(x-\xi)}{\partial x_{1}}\right|_{\xi_{2}=\gamma_{1}\left(\xi_{1}\right)} d x, \\
& \left.\frac{1}{2} \frac{\partial u(\xi)}{\partial \xi_{1}}\right|_{\xi_{2}=\gamma_{2}\left(\xi_{1}\right)} \\
& =-\left.\frac{1}{2 \pi} \int_{a_{1}}^{b_{1}} \frac{\partial u(x)}{\partial x_{1}}\right|_{x_{2}=\gamma_{1}\left(x_{1}\right)} \frac{\gamma_{1}\left(x_{1}\right)-\gamma_{2}\left(\xi_{1}\right)+\left(x_{1}-\xi_{1}\right) \gamma_{1}^{\prime}\left(x_{1}\right)}{\left(x_{1}-\xi_{1}\right)^{2}+\left[\gamma_{1}\left(x_{1}\right)-\gamma_{2}\left(\xi_{1}\right)\right]^{2}} d x_{1} \\
& +\left.\frac{1}{2 \pi} \int_{a_{1}}^{b_{1}} \frac{\partial u(x)}{\partial x_{1}}\right|_{x_{2}=\gamma_{2}\left(x_{1}\right)} \frac{\gamma_{2}^{\prime}\left(\sigma_{2}\right)-\gamma_{2}^{\prime}\left(x_{1}\right)}{1+\gamma_{2}^{\prime 2}\left(\sigma_{2}\right)} \frac{d x_{1}}{x_{1}-\xi_{1}} \\
& -\left.\frac{1}{2 \pi} \int_{a_{1}}^{b_{1}} \frac{\partial u(x)}{\partial x_{2}}\right|_{x_{2}=\gamma_{1}\left(x_{1}\right)} \frac{x_{1}-\xi_{1}+\left[\gamma_{1}\left(x_{1}\right)-\gamma_{2}\left(\xi_{1}\right)\right] \gamma_{1}^{\prime}(x)}{\left(x_{1}-\xi_{1}\right)^{2}+\left[\gamma_{1}\left(x_{1}\right)-\gamma_{2}\left(\xi_{1}\right)\right]^{2}} d x_{1}
\end{aligned}
$$




$$
\begin{aligned}
& -\left.\frac{1}{2 \pi} \int_{a_{1}}^{b_{1}} \frac{\partial u(x)}{\partial x_{2}}\right|_{x_{2}=\gamma_{2}\left(x_{1}\right)} \frac{1+\gamma_{2}^{\prime}\left(\sigma_{2}\right) \gamma_{2}^{\prime}\left(x_{1}\right)}{1+\gamma_{2}^{\prime 2}\left(\sigma_{2}\right)} \frac{d x_{1}}{x_{1}-\xi_{1}} \\
& +\left.\int_{D} \sum_{j=1}^{2} a_{j}(x) \frac{\partial u(x)}{\partial x_{j}} \frac{\partial U(x-\xi)}{\partial x_{1}}\right|_{\xi_{2}=\gamma_{2}\left(\xi_{1}\right)} d x \\
& +\left.\int_{D} a_{0}(x) u(x) \frac{\partial U(x-\xi)}{\partial x_{1}}\right|_{\xi_{2}=\gamma_{2}\left(\xi_{1}\right)} d x \\
& +\int_{D}\left\{\left.\int_{a_{1}}^{b_{1}} \sum_{n=1}^{2}\left[\sum_{j=1}^{2} K_{j n}\left(x, \eta_{1}\right) \frac{\partial u(\eta)}{\partial \eta_{j}}+K_{0 n}\left(x, \eta_{1}\right) u(\eta)\right]\right|_{\eta_{2}=\gamma_{n}\left(\eta_{1}\right)} d \eta_{1}\right. \\
& \left.+\int_{D}\left[\sum_{j=1}^{2} K_{j}(x, \eta) \frac{\partial u(\eta)}{\partial \eta_{j}}+K_{0}(x, \eta) u(\eta)\right] d \eta\right\}\left.\frac{\partial U(x-\xi)}{\partial x_{1}}\right|_{\xi_{2}=\gamma_{2}\left(\xi_{1}\right)} d x \\
& -\left.\int_{D} f(x) \frac{\partial U(x-\xi)}{\partial x_{1}}\right|_{\xi_{2}=\gamma_{2}\left(\xi_{1}\right)} d x,
\end{aligned}
$$

$\frac{1}{2} \frac{\partial u(\xi)}{\partial \xi_{2}}$

$$
\begin{aligned}
= & -\left.\frac{1}{2 \pi} \int_{a_{1}}^{b_{1}} \frac{\partial u(x)}{\partial x_{1}}\right|_{x_{2}=\gamma_{1}\left(x_{1}\right)} \frac{1+\gamma_{1}^{\prime}\left(\sigma_{1}\right) \gamma_{1}^{\prime}\left(x_{1}\right)}{1+\gamma_{1}^{\prime 2}\left(\sigma_{1}\right)} \frac{d x_{1}}{x_{1}-\xi_{1}} \\
& +\left.\frac{1}{2 \pi} \int_{a_{1}}^{b_{1}} \frac{\partial u(x)}{\partial x_{1}}\right|_{x_{2}=\gamma_{2}\left(x_{1}\right)} \frac{x_{1}-\xi_{1}+\left[\gamma_{2}\left(x_{1}\right)-\gamma_{1}\left(\xi_{1}\right)\right] \gamma_{2}^{\prime}\left(x_{1}\right)}{\left(x_{1}-\xi_{1}\right)^{2}+\left[\gamma_{2}\left(x_{1}\right)-\gamma_{1}\left(\xi_{1}\right)\right]^{2}} d x_{1} \\
& -\left.\frac{1}{2 \pi} \int_{a_{1}}^{b_{1}} \frac{\partial u(x)}{\partial x_{2}}\right|_{x_{2}=\gamma_{1}\left(x_{1}\right)} \frac{\gamma_{1}^{\prime}\left(\sigma_{1}\right)-\gamma_{1}^{\prime}\left(x_{1}\right)}{x_{1}-\xi_{1}} \frac{d x_{1}}{1+\gamma_{1}^{\prime 2}\left(\sigma_{1}\right)} \\
& +\left.\frac{1}{2 \pi} \int_{a_{1}}^{b_{1}} \frac{\partial u(x)}{\partial x_{2}}\right|_{x_{2}=\gamma_{2}\left(x_{1}\right)} \frac{\gamma_{2}\left(x_{1}\right)-\gamma_{1}\left(\xi_{1}\right)+\left(x_{1}-\xi_{1}\right) \gamma_{2}^{\prime}\left(x_{1}\right)}{\left(x_{1}\right)^{2}+\left[\gamma_{2}\left(x_{1}\right)-\gamma_{1}\left(\xi_{1}\right)\right]^{2}} d x_{1} \\
& +\left.\int_{D} \sum_{j=1}^{2} a_{j}(x) \frac{\partial u(x)}{\partial x_{j}} \frac{\partial U(x-\xi)}{\partial x_{2}}\right|_{\xi_{2}=\gamma_{1}\left(\xi_{1}\right)} ^{d x} d x \\
& +\left.\int_{D} a(x) u(x) \frac{\partial U(x-\xi)}{\partial x_{2}}\right|_{\xi_{2}=\gamma_{1}\left(\xi_{1}\right)} d x \\
& +\int_{D}\left\{\left.\int_{a_{1}}^{b_{1}} \sum_{n=1}^{2}\left[\sum_{j=1}^{2} K_{j n}\left(x, \eta_{1}\right) \frac{\partial u(\eta)}{\partial \eta_{j}}+K_{0 n}\left(x, \eta_{1}\right) u(\eta)\right]\right|_{\eta_{2}=\gamma_{n}\left(\eta_{1}\right)} d \eta_{1}\right.
\end{aligned}
$$


Abstract and Applied Analysis

$$
\begin{aligned}
& \left.+\int_{D}\left[\sum_{j=1}^{2} K_{j}(x, \eta) \frac{\partial u(\eta)}{\partial \eta_{j}}+K_{0}(x, \eta) u(\eta)\right] d \eta\right\}\left.\frac{\partial U(x-\xi)}{\partial x_{2}}\right|_{\xi_{2}=\gamma_{1}\left(\xi_{1}\right)} d x \\
& -\left.\int_{D} f(x) \frac{\partial U(x-\xi)}{\partial x_{2}}\right|_{\xi_{2}=\gamma_{1}\left(\xi_{1}\right)} d x .
\end{aligned}
$$

$\left.\frac{1}{2} \frac{\partial u(\xi)}{\partial \xi_{2}}\right|_{\xi_{2}=\gamma_{2}\left(\xi_{1}\right)}$

$$
\begin{aligned}
& =\left.\frac{1}{2 \pi} \int_{a_{1}}^{b_{1}} \frac{\partial u(x)}{\partial x_{1}}\right|_{x_{2}=\gamma_{1}\left(x_{1}\right)} \frac{x_{1}-\xi_{1}+\left[\gamma_{1}\left(x_{1}\right)-\gamma_{2}\left(\xi_{1}\right)\right] \gamma_{1}^{\prime}\left(x_{1}\right)}{\left(x_{1}-\xi_{1}\right)^{2}+\left[\gamma_{1}\left(x_{1}\right)-\gamma_{2}\left(\xi_{1}\right)\right]^{2}} d x_{1} \\
& +\left.\frac{1}{2 \pi} \int_{a_{1}}^{b_{1}} \frac{\partial u(x)}{\partial x_{1}}\right|_{x_{2}=\gamma_{2}\left(x_{1}\right)} \frac{1+\gamma_{2}^{\prime}\left(\sigma_{2}\right) \gamma_{2}^{\prime}\left(x_{1}\right)}{1+\gamma_{2}^{\prime 2}\left(\sigma_{2}\right)} \frac{d x_{1}}{x_{1}-\xi_{1}} \\
& -\left.\frac{1}{2 \pi} \int_{a_{1}}^{b_{1}} \frac{\partial u(x)}{\partial x_{2}}\right|_{x_{2}=\gamma_{1}\left(x_{1}\right)} \frac{\gamma_{1}\left(x_{1}\right)-\gamma_{2}\left(\xi_{1}\right)-\left(x_{1}-\xi_{1}\right) \gamma_{1}^{\prime}\left(x_{1}\right)}{\left(x_{1}-\xi_{1}\right)^{2}+\left[\gamma_{1}\left(x_{1}\right)-\gamma_{2}\left(\xi_{1}\right)\right]^{2}} d x_{1} \\
& +\left.\frac{1}{2 \pi} \int_{a_{1}}^{b_{1}} \frac{\partial u(x)}{\partial x_{2}}\right|_{x_{2}=\gamma_{2}\left(x_{1}\right)} \frac{\gamma_{2}^{\prime}\left(\sigma_{2}\right)-\gamma_{2}^{\prime}\left(x_{1}\right)}{1+\gamma_{2}^{\prime 2}\left(\sigma_{2}\right)} \frac{d x_{1}}{x_{1}-\xi_{1}} \\
& +\left.\int_{D} \sum_{j=1}^{2} a_{j}(x) \frac{\partial u(x)}{\partial x_{j}} \frac{\partial U(x-\xi)}{\partial x_{2}}\right|_{\xi_{2}=\gamma_{2}\left(\xi_{1}\right)} d x \\
& +\left.\int_{D} a_{0}(x) u(x) \frac{\partial U(x-\xi)}{\partial x_{2}}\right|_{\xi_{2}=\gamma_{2}\left(\xi_{1}\right)} d x \\
& +\int_{D}\left\{\left.\int_{a_{1}}^{b_{1}} \sum_{n=1}^{2}\left[\sum_{j=1}^{2} K_{j n}\left(x, \eta_{1}\right) \frac{\partial u(\eta)}{\partial \eta_{j}}+K_{0 n}\left(x, \eta_{1}\right) u(\eta)\right]\right|_{\eta_{2}=\gamma_{n}\left(\eta_{1}\right)} d \eta_{1}\right. \\
& \left.+\int_{D}\left[\sum_{j=1}^{2} K_{j}(x, \eta) \frac{\partial u(\eta)}{\partial \eta_{j}}+K_{0}(x, \eta) u(\eta)\right] d \eta\right\}\left.\frac{\partial U(x-\xi)}{\partial x_{2}}\right|_{\xi_{2}=\gamma_{2}\left(\xi_{1}\right)} d x \\
& -\left.\int_{D} f(x) \frac{\partial U(x-\xi)}{\partial x_{2}}\right|_{\xi_{2}=\gamma_{2}\left(\xi_{1}\right)} d x .
\end{aligned}
$$

Thus, we prove the following statement.

Theorem 5.2. Under the conditions of Theorem 4.1, each of the necessary conditions (5.7), (5.8), (5.9), and (5.10) contains one singular term. 


\section{Separation of Singularities}

As it was noted in Theorem 5.2, the last four of six necessary conditions have singularities.

Let us separate these singularities, that is, determine their coefficients. It is easy to check that

$$
\frac{1+\gamma_{k}^{\prime}\left(\sigma_{k}\right) \gamma_{k}^{\prime}\left(x_{1}\right)}{1+\gamma_{k}^{\prime 2}\left(\sigma_{k}\right)}=1+\left[\frac{1+\gamma_{k}^{\prime}\left(\sigma_{k}\right) \gamma_{k}^{\prime}\left(x_{1}\right)}{1+\gamma_{k}^{\prime 2}\left(\sigma_{k}\right)}-1\right]=1+\frac{\gamma_{k}^{\prime}\left(\sigma_{k}\right)\left[\gamma_{k}^{\prime}\left(x_{1}\right)-\gamma_{k}^{\prime}\left(\sigma_{k}\right)\right]}{1+\gamma_{k}^{\prime 2}\left(\sigma_{k}\right)}, \quad k=1,2 .
$$

Therefore, we write the necessary conditions in theorem 3 in the following form, where pure singular terms are noted

$$
\begin{aligned}
& \left.\frac{\partial u(\xi)}{\partial \xi_{1}}\right|_{\xi_{2}=\gamma_{k}\left(\xi_{1}\right)}=-\left.\frac{1}{\pi} \int_{a_{1}}^{b_{1}} \frac{\partial u(x)}{\partial x_{2}}\right|_{x_{2}=\gamma_{k}\left(x_{1}\right)} \frac{d x_{1}}{x_{1}-\xi_{1}}+\ldots, \quad k=1,2, \\
& \left.\frac{\partial u(\xi)}{\partial \xi_{2}}\right|_{\xi_{2}=\gamma_{k}\left(\xi_{1}\right)}=\left.\frac{1}{\pi} \int_{a_{1}}^{b_{1}} \frac{\partial u(x)}{\partial x_{1}}\right|_{x_{2}=\gamma_{k}\left(x_{1}\right)} \frac{d x_{1}}{x_{1}-\xi_{1}}+\ldots, \quad k=1,2 .
\end{aligned}
$$

The dots denote the sum of nonsingular terms.

\section{Fredholm Property}

Considering necessary conditions (6.2), we construct the following linear combination:

$$
\begin{aligned}
\sum_{n=1}^{2}[ & \left.-\left.\alpha_{m 2 n}\left(\xi_{1}\right) \frac{\partial u(\xi)}{\partial \xi_{1}}\right|_{\xi_{2}=\gamma_{n}\left(\xi_{1}\right)}+\left.\alpha_{m 1 n}\left(\xi_{1}\right) \frac{\partial u(\xi)}{\partial \xi_{2}}\right|_{\xi_{2}=\gamma_{n}\left(\xi_{1}\right)}\right] \\
& =\left.\frac{1}{\pi} \int_{a_{1}}^{b_{1}} \sum_{n=1}^{2} \sum_{j=1}^{2} \alpha_{m j n}\left(\xi_{1}\right) \frac{\partial u(x)}{\partial x_{j}}\right|_{x_{2}=\gamma_{n}\left(x_{1}\right)} \frac{d x_{1}}{x_{1}-\xi_{1}}+\ldots, \quad m=1,2 .
\end{aligned}
$$

To the right-hand side under the sign of integral we substitute their expression from boundary conditions (2.2). Considering the schemes of $[8,9]$, we obtain the following:

$$
\begin{aligned}
\sum_{n=1}^{2}\left[-\left.\alpha_{m 2 n}\left(\xi_{1}\right) \frac{\partial u(\xi)}{\partial \xi_{1}}\right|_{\xi_{2}=\gamma_{n}\left(\xi_{1}\right)}+\left.\alpha_{m 1 n}\left(\xi_{1}\right) \frac{\partial u(\xi)}{\partial \xi_{2}}\right|_{\xi_{2}=\gamma_{n}\left(\xi_{1}\right)}\right] \\
=\frac{1}{\pi} \int_{a_{1}}^{b_{1}}\left\{-\left.\sum_{n=1}^{2} \alpha_{m 0 n}\left(x_{1}\right) u(x)\right|_{x_{2}=\gamma_{n}\left(x_{1}\right)}\right. \\
-\left.\int_{a_{1}}^{b_{1}} \sum_{n=1}^{2}\left[\sum_{j=1}^{2} A_{m j k}\left(x_{1}, \eta_{1}\right) \frac{\partial u(\eta)}{\partial \eta_{j}}+A_{m 0 k}\left(x_{1}, \eta_{1}\right) u(\eta)\right]\right|_{\eta_{2}=\gamma_{n}\left(\eta_{1}\right)} d \eta_{1} \\
\left.-\int_{D}\left[\sum_{j=1}^{2} A_{m j}\left(x_{1}, \eta\right) \frac{\partial u(\eta)}{\partial \eta_{j}}+A_{m 0}\left(x_{1}, \eta\right) u(\eta)\right] d \eta+\alpha_{m}\left(x_{1}\right)\right\} \\
\times \frac{d x_{1}}{x_{1}-\xi_{1}}+\cdots, \quad m=1,2 .
\end{aligned}
$$

Thus, we proved the following. 
Theorem 7.1. Under the conditions of theorem 1, if boundary conditions (2.2) are linear independent, the coefficients $\alpha_{m j n}\left(x_{1}\right), m, j$, and $n=1,2$ belong to some Holder class, all the kernels of the integrals in boundary conditions (2.2) are continuous, and the right-hand side $\alpha_{m}\left(x_{1}\right)$ for $m=1,2$ are continuously differentiable functions vanishing at the end of the interval $\left(a_{1}, b_{1}\right)$, then relations (7.2) are regular.

Really, for regularization of the first term in the right-hand side of (7.2), it suffices to consider regular relations (5.5) and (5.6) for $u\left(x_{1}, \gamma_{1}\left(x_{1}\right)\right), u\left(x_{1}, \gamma_{2}\left(x_{1}\right)\right)$, and interchange integrals after substitution.

The last term does not contain unknown functions and is understood in the sense of the principal value and is regularized in (7.2). All intermediate terms are regularized by permutation of integrals contained in these terms.

Finally, combining (2.2) and (7.2), from the obtained four relations we arrive at the following restriction:

$$
\left|\begin{array}{cccc}
\alpha_{111}\left(\xi_{1}\right) & \alpha_{112}\left(\xi_{1}\right) & \alpha_{121}\left(\xi_{1}\right) & \alpha_{122}\left(\xi_{1}\right) \\
\alpha_{211}\left(\xi_{1}\right) & \alpha_{212}\left(\xi_{1}\right) & \alpha_{221}\left(\xi_{1}\right) & \alpha_{222}\left(\xi_{1}\right) \\
-\alpha_{121}\left(\xi_{1}\right) & -\alpha_{122}\left(\xi_{1}\right) & \alpha_{111}\left(\xi_{1}\right) & \alpha_{112}\left(\xi_{1}\right) \\
-\alpha_{221}\left(\xi_{1}\right) & -\alpha_{222}\left(\xi_{1}\right) & \alpha_{211}\left(\xi_{1}\right) & \alpha_{212}\left(\xi_{1}\right)
\end{array}\right| \neq 0,
$$

that is, a sufficient condition for reduction of (2.2), (7.2) with respect to boundary conditions to $\partial u(x) / \partial x_{11}$ and $\partial u(x) / \partial x_{2}$ the normal form.

Thus, combining (5.5), (5.6), (2.2), and (7.2), we get (for boundary values) for $u(x)$, $\partial u(x) / \partial x_{1}$, and $\partial u(x) / \partial x_{2}$ six second type Fredholm regular integral equations. Finally, considering three integral equations for $u(x), \partial u(x) / \partial x_{1}$, and $\partial u(x) / \partial x_{2}$ for $x \in D$, obtained from the first expressions of principal relations (4.1)-(4.3), we get the following result.

Theorem 7.2. Under the conditions of Theorem 7.1 and condition (7.3), boundary value problem (2.1)-(2.2) is of Fredholm type.

\section{References}

[1] S. L. Sobolev, Equations of Mathematical Physics, Nauka, Russia, 4th edition, 1966.

[2] A. N. Tikhonov and A. A. Samarskii, Equations of Mathematical Physics, Nauka, Russia, 1967.

[3] I. G. Petrovski1̌, Lectures on Partial Equations, Nauka, Russia, 1970.

[4] V. P. Mijailov, Partial Differential Equations, Mir, Moscow, Russia, 1978.

[5] F. G. Toricomi, Differential Equations, Blacxie \& Son limited, Glasgow, Scotland, 1961.

[6] R. Courant, Partial Differential Equations, Wiley-Interscience, New York, NY, USA, 1962.

[7] G. Kavei and N. Aliev, "An analytical method to the solution of the time-dependent Schrodinger equation using half cylinder space system. I," Bulletin of Pure $\mathcal{E}$ Applied Sciences, vol. 16, no. 2, pp. 253-263, 1997.

[8] N. Aliev and M. Jahanshahi, "Solution of Poisson's equation with global, local and nonlocal boundary conditions," International Journal of Mathematical Education in Science and Technology, vol. 33, no. 2, pp. 241-247, 2002.

[9] F. Bahrami, N. Aliev, and S. M. Hosseini, "A method for the reduction of four dimensional mixed problems with general boundary conditions to a system of second kind Fredholm integral equations," Italian Journal of Pure and Applied Mathematics, no. 17, pp. 91-104, 2005.

[10] V. S. Vladimirov, Equations of Mathematical Physics, Mir, Moscow, Russia, 1984. 
[11] N. Aliev and S. M. Hosseini, "A regularization of Fredholm type singular integral equations," International Journal of Mathematics and Mathematical Sciences, vol. 26, no. 2, pp. 123-128, 2001.

[12] N. Aliev and S. M. Hosseini, "Multidimensional singular Fredholm integral equations in a finite domain and their regularization," Southeast Asian Bulletin of Mathematics, vol. 27, no. 3, pp. 395-408, 2003. 


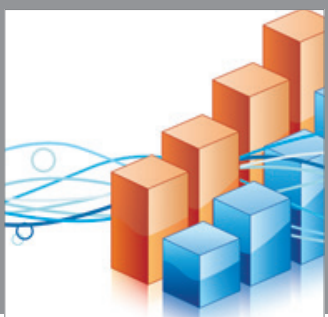

Advances in

Operations Research

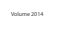

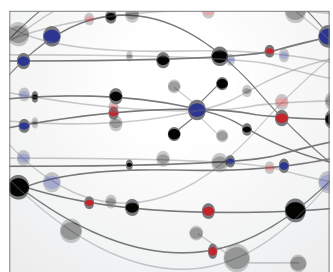

\section{The Scientific} World Journal
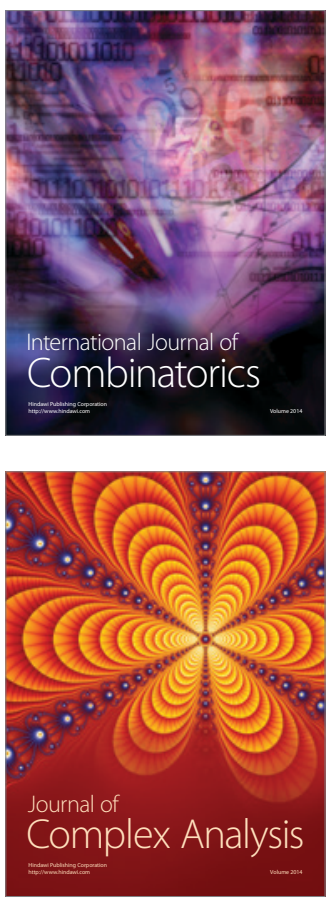

International Journal of

Mathematics and

Mathematical

Sciences
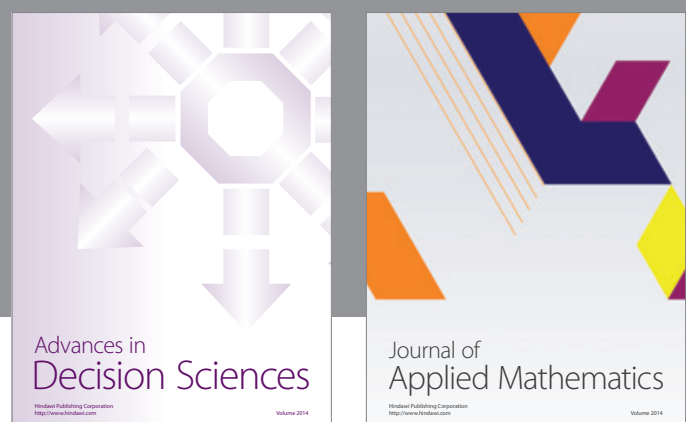

Journal of

Applied Mathematics
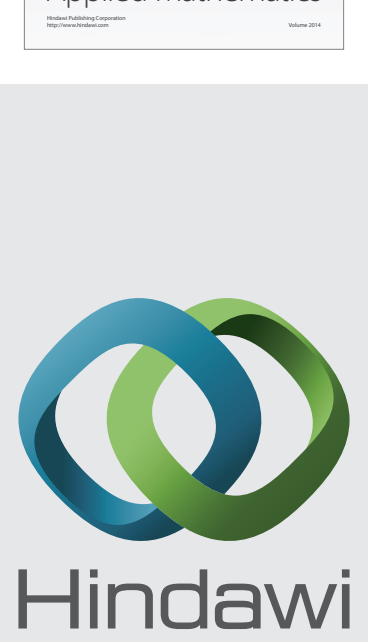

Submit your manuscripts at http://www.hindawi.com
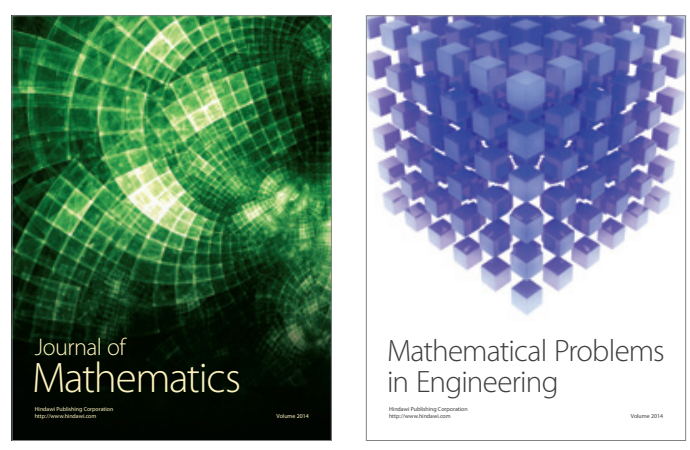

Mathematical Problems in Engineering
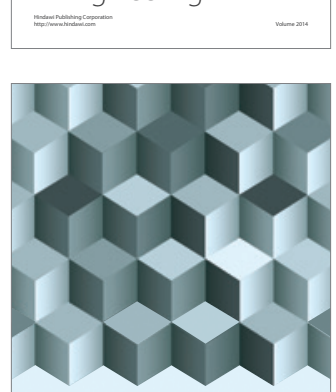

Journal of

Function Spaces
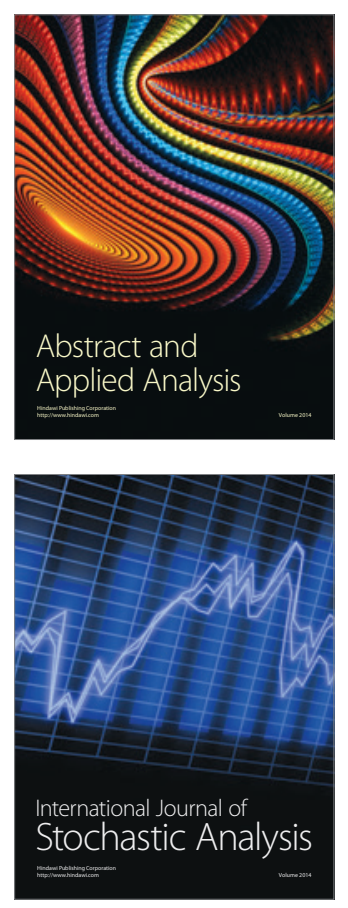

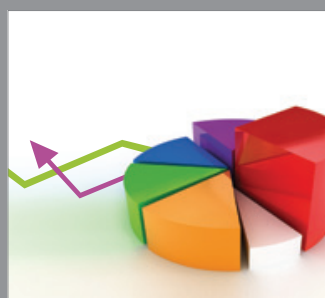

ournal of

Probability and Statistics

Promensencen
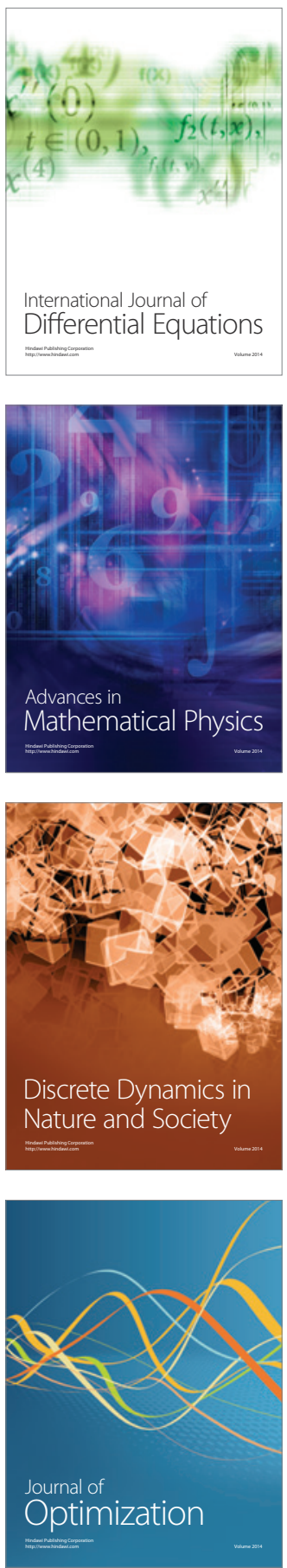